\title{
SMOOTH VECTORS FOR HIGHEST WEIGHT REPRESENTATIONS
}

\author{
KARL-HERMANN NEEB \\ Fachbereich Mathematik, TU Darmstadt, Schlossgartenstrasse 7, D-64289 Darmstadt, Germany \\ e-mail:neeb@mathematik.tu-darmstadt.de
}

(Received 10 February, 1999)

\begin{abstract}
Let $\left(\pi_{\lambda}, \mathcal{H}_{\lambda}\right)$ be a unitary highest weight representation of the connected Lie group $G$ and $g$ its Lie algebra. Then $g$ contains an invariant closed convex cone $W_{\max }$ such that, for each $X \in W_{\max }^{0}$, the selfadjoint operator $i \cdot d \pi_{\lambda}(X)$ is bounded from above. We show that for each such $X$, the space $\mathcal{H}_{\lambda}^{\infty}$ of smooth vectors for the action of $G$ on $\mathcal{H}_{\lambda}$ coincides with the set $\mathcal{D}^{\infty}\left(d \pi_{\lambda}(X)\right)$ of smooth vectors for the generally unbounded operator $d \pi_{\lambda}(X)$.
\end{abstract}

1991 Mathematics Subject Classification. Primary 17B15, Secondary 20 E45.

0. Introduction. Let $\left(\pi_{\lambda}, \mathcal{H}_{\lambda}\right)$ be a unitary highest weight representation of the finite-dimensional connected Lie group $G$ and $g$ its Lie algebra. Then $g$ contains an invariant closed convex cone $W_{\max }$ such that, for each $X \in W_{\max }^{0}$, the selfadjoint operator $i \cdot d \pi_{\lambda}(X)$ is bounded from above. In this note we show that, for each such $X$, the space $\mathcal{H}_{\lambda}^{\infty}$ of smooth vectors for the action of $G$ on $\mathcal{H}_{\lambda}$ coincides with the set $\mathcal{D}^{\infty}\left(d \pi_{\lambda}(X)\right)$ of smooth vectors for the generally unbounded operator $d \pi_{\lambda}(X)$. This result greatly facilitates the determination of the space of smooth vectors and hence of the distribution vectors for highest weight representations.

Our result has been motivated by the following problem. Suppose that we have realized the representation $\left(\pi_{\lambda}, \mathcal{H}_{\lambda}\right)$ in a space of holomorphic functions on a complex domain $\mathcal{D}$ with values in some finite dimensional complex vector space $V$ (cf. [1], [2], [3], [4] and [12, Chapter XII]). Then the action of $G$ and of its Lie algebra $g$ extend naturally to the space $\operatorname{Hol}(\mathcal{D}, V)$ of all holomorphic $V$-valued functions on $\mathcal{D}$. Now the characterization from above implies, in particular, that the space $\mathcal{H}_{\lambda}^{-\infty}$ of distribution vectors can be described as $\sum_{n \in \mathbb{N}} d \pi_{\lambda}(X)^{n} . \mathcal{H}_{\lambda}$. If, in addition, $X$ is contained in the center of a maximal compactly embedded subalgebra, then the action of $X$ on $\operatorname{Hol}(\mathcal{D}, V)$ is quite simply given by some sort of Euler operator and the result described in this paper can be used to obtain a direct analytic characterization of the elements of the space $\mathcal{H}_{\lambda}^{-\infty}$ considered as a space of $V$-valued holomorphic functions on $\mathcal{D}$.

The description of the smooth and therefore the distribution vectors of a highest weight representation is an essential step towards the determination of the $H$-invariant distribution vectors for a given closed subgroup $H$ of $G$. A question that is particularly interesting, and still open, is the case in which $(G, H)$ is a symmetric pair.

\section{Basic concepts and definitions.}

Definition 1.1. (a) Let $g$ be a finite dimensional real Lie algebra. For a subalgebra $a \subseteq \mathfrak{g}$ we write $\operatorname{Inn}(\mathfrak{a}):=\left\langle e^{\text {ad } \mathfrak{}}\right\rangle \subseteq \operatorname{Aut}(\mathfrak{g})$ for the corresponding group of 
inner automorphisms. A subalgebra $\mathfrak{a} \subseteq \mathfrak{g}$ is said to be compactly embedded if $\operatorname{Inn}(\mathfrak{a})$ has compact closure.

(b) Let $\mathrm{t} \subseteq \mathrm{g}$ be a compactly embedded Cartan subalgebra and recall that there exists a unique maximal compactly embedded subalgebra $\mathfrak{f}$ containing $t$ (cf. [5, A.2.40]).

(c) Associated to the Cartan subalgebra $t_{\mathbb{C}}$ in the complexification $\mathfrak{g}_{\mathbb{C}}$ is a root decomposition as follows. For a linear functional $\alpha \in \mathfrak{t}_{\mathbb{C}}^{*}$ we set

$$
\mathfrak{g}_{\mathbb{C}}^{\alpha}:=\left\{X \in \mathfrak{g}_{\mathbb{C}}:\left(\forall Y \in \mathfrak{t}_{\mathbb{C}}\right)[Y, X]=\alpha(Y) X\right\}
$$

and we shall write $\Delta:=\left\{\alpha \in \mathfrak{t}_{\mathbb{C}}^{*} \backslash\{0\}: \mathfrak{g}_{\mathbb{C}}^{\alpha} \neq\{0\}\right\}$ for the set of roots. Then $\mathfrak{g}_{\mathbb{C}}=\mathfrak{t}_{\mathbb{C}} \oplus \bigoplus_{\alpha \in \Delta} \mathfrak{g}_{\mathbb{C}}^{\alpha}, \alpha(\mathfrak{t}) \subseteq i \mathbb{R}$, for all $\alpha \in \Delta$, and $\overline{\mathfrak{g}_{\mathbb{C}}^{\alpha}}=\mathfrak{g}_{\mathbb{C}}^{-\alpha}$, where $X \mapsto \bar{X}$ denotes complex conjugation on $\mathfrak{g}_{\mathbb{C}}$ with respect to $\mathfrak{g}$. Put $X^{*}:=-\bar{X}$

(d) A root $\alpha$ is said to be compact if $\mathfrak{g}_{\mathbb{C}}^{\alpha} \subseteq \mathfrak{f}_{\mathbb{C}}$ and non-compact otherwise. We write $\Delta_{k}$ for the set of compact and $\Delta_{p}$ for the set of non-compact roots. If $\mathfrak{g}=\mathfrak{r} \rtimes \mathfrak{g}$ is a $\mathfrak{f}$-invariant Levi decomposition, then we set

$$
\Delta_{r}:=\left\{\alpha \in \Delta: \mathfrak{g}_{\mathbb{C}}^{\alpha} \subseteq \mathfrak{r}_{\mathbb{C}}\right\} \quad \text { and } \quad \Delta_{s}:=\left\{\alpha \in \Delta: \mathfrak{g}_{\mathbb{C}}^{\alpha} \subseteq \mathfrak{S}_{\mathbb{C}}\right\}
$$

Recall that $\Delta=\Delta_{r} \dot{\cup} \Delta_{S}$ is a disjoint decomposition (cf. [12, Definition VII.2.4]). If $\alpha \in \Delta_{S}$, then we write $\check{\alpha}$ for the uniquely determined element in the one-dimensional space $\left[\mathfrak{g}_{\mathbb{C}}^{\alpha}, \mathfrak{g}_{\mathbb{C}}^{-\alpha}\right] \subseteq \mathfrak{t}_{\mathbb{C}}$ satisfying $\alpha(\check{\alpha})=2$.

(e) A positive system $\Delta^{+}$of roots is a subset of $\Delta$ for which there exists a regular element $X_{0} \in$ it with $\Delta^{+}=\left\{\alpha \in \Delta: \alpha\left(X_{0}\right)>0\right\}$. We say that a positive system $\Delta^{+}$is $\mathfrak{f}$ adapted if the set $\Delta_{p}^{+}:=\Delta_{p} \cap \Delta^{+}$of positive non-compact roots is invariant under the Weyl group $\mathcal{W}_{\mathfrak{f}}:=N_{\operatorname{Inn}(\mathfrak{f})}(\mathrm{t}) / Z_{\operatorname{Inn}(\mathfrak{f})}(\mathrm{t})$ acting on $\mathrm{t}$. We recall from $[\mathbf{8}$, Proposition II.7] (cf. also [12, Proposition VII.2.14]) that there exists a $f$-adapted positive system if and only if $z_{\mathfrak{g}}(z(\mathfrak{f}))=\mathfrak{f}$. In this case we say that $\mathfrak{g}$ is quasihermitian. Note that a simple real Lie algebra is quasihermitian if and only if it is either compact or hermitian.

(f) We associate to a positive system $\Delta^{+}$the convex cones

$$
C_{\min }:=\operatorname{cone}\left\{i\left[\overline{X_{\alpha}}, X_{\alpha}\right]: X_{\alpha} \in \mathfrak{g}_{\mathbb{C}}^{\alpha}, \alpha \in \Delta_{p}^{+}\right\}
$$

and $C_{\max }:=\left(i \Delta_{p}^{+}\right)^{\star}=\left\{X \in \mathrm{t}:\left(\forall \alpha \in \Delta_{p}^{+}\right) i \alpha(X) \geq 0\right\}$.

In the following we assume that $g$ is a quasihermitian Lie algebra, $t \subseteq g$ is a compactly embedded Cartan subalgebra, and $\Delta$ is the corresponding system of roots.

Definition 1.2. Let $\Delta^{+} \subseteq \Delta$ be a positive system.

(a) For a $\mathfrak{g}_{\mathbb{C}}$-module $V$ and $\beta \in \mathfrak{t}_{\mathbb{C}}^{*}$ we set $V^{\beta}:=\left\{v \in V:\left(\forall X \in \mathfrak{t}_{\mathbb{C}}\right) X . v=\beta(X) v\right\}$. This space is called the weight space of weight $\beta$ and $\beta$ is said to be a weight of $V$ if $V^{\beta} \neq\{0\}$. We write $\mathcal{P}_{V}$ for the set of weights of $V$.

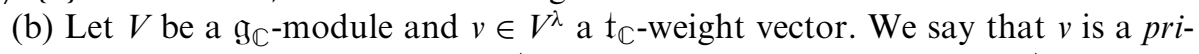
mitive element of $V$ (with respect to $\Delta^{+}$) if $g_{\mathbb{C}}^{\alpha} \cdot v=\{0\}$ holds for all $\alpha \in \Delta^{+}$.

(c) $\mathrm{A} \mathfrak{g}_{\mathbb{C}^{-}}$-module $V$ is called a highest weight module with highest weight $\lambda$ (with respect to $\Delta^{+}$) if it is generated by a primitive element of weight $\lambda$.

(d) A highest weight module $V$ is said to be unitarizable if there exists a unitary representation $(\pi, \mathcal{H})$ of the simply connected Lie group $G$ with $\mathbf{L}(G)=\mathfrak{g}$ such that 
$V$ is isomorphic to the $\mathfrak{g}_{\mathbb{C}}$-module $\mathcal{H}^{K}$ of $K$-finite vectors in $\mathcal{H}$ (cf. [8, Section III]), where $K=\exp f$. In this case $(\pi, \mathcal{H})$ is called a unitary highest weight representation of $G$.

2. Estimates and smooth vectors for highest weight representations. Let $L(\lambda)$ be a unitary highest weight module with respect to $\Delta^{+}$and highest weight $\lambda$. We recall from [10] that such a highest weight module exists essentially only for $\mathfrak{f}$-adapted positive systems).

We write $\mathcal{H}_{\lambda}$ for the Hilbert space completion of $L(\lambda)$ and $\pi_{\lambda}$ for the unitary representation of the corresponding simply connected group $G$ on $\mathcal{H}_{\lambda}$. If $\mathcal{H}_{\lambda}^{\omega}$ denotes the subspace of analytic vectors of the representation $\left(\pi_{\lambda}, \mathcal{H}_{\lambda}\right)$, then $L(\lambda) \hookrightarrow \mathcal{H}^{\omega}$ is an embedding of $\mathfrak{g}_{\mathbb{C}}$-modules. As the theory of holomorphic representations shows, the representation of $G$ on $\mathcal{H}_{\lambda}$ extends to a holomorphic representation of the maximal Ol'shanskii semigroup $S=G \exp \left(i W_{\text {max }}^{0}\right)$ on $\mathcal{H}_{\lambda}$ (cf. [10, Section 4] or [12, Chapter XI]). Let $X_{0} \in i W_{\max }^{0}$. Then [7, Proposition A.5] shows that

$$
\mathcal{H}^{\omega}=\bigcup_{t>0} \pi_{\lambda}\left(\exp t X_{0}\right) \cdot \mathcal{H}
$$

From the representation theory of the group $\mathbb{R}$ one now gets

$$
\mathcal{H}^{\omega}=\mathcal{D}^{\omega}\left(d \pi\left(X_{0}\right)\right)=\left\{v \in \mathcal{H}:(\exists t>0) \sum_{n=0}^{\infty} \frac{t^{n}}{n !}\left\|d \pi\left(X_{0}\right)^{n} . v\right\|<\infty\right\}
$$

that is, $\mathcal{H}^{\omega}$ coincides with the space of analytic vectors for the selfadjoint operator $d \pi\left(X_{0}\right)$.

In this note we shall see that this characterization carries over to the space of smooth vectors. We shall show that

$$
\mathcal{H}^{\infty}=\mathcal{D}^{\infty}\left(d \pi\left(X_{0}\right)\right):=\bigcap_{n \in \mathbb{N}} \mathcal{D}\left(d \pi\left(X_{0}\right)^{n}\right)
$$

Both characterizations yield quite explicit descriptions of the space of smooth and analytic vectors in terms of the weight decomposition. Let $\mathcal{P}_{\lambda}:=\mathcal{P}_{L(\lambda)} \subseteq \mathrm{t}_{\mathbb{C}}^{*}$ denote the set of weights of the $\mathfrak{g}_{\mathbb{C}}$-module $L(\lambda), L(\lambda)=\sum_{\beta \in \mathcal{P}_{\lambda}} L(\lambda)^{\beta}$ the corresponding weight space decomposition, and write $v=\sum_{\alpha} v_{\alpha}$ for the corresponding decomposition of an element of $v \in \mathcal{H}_{\lambda}$. We note that $\|v\|^{2}=\sum_{\alpha}\left\|v_{\alpha}\right\|^{2}$. Since $W_{\text {max }}^{0}=\operatorname{Ad}(G) .\left(W_{\max }^{0} \cap \mathrm{t}\right)(\mathrm{cf}$. [11, Lemma II.10]), we may without loss of generality assume that $X_{0} \in i\left(\mathrm{t} \cap W_{\max }^{0}\right)=i C_{\max }^{0}$. Then

$$
d \pi\left(X_{0}\right) \cdot v=\sum_{\alpha} \alpha\left(X_{0}\right) v_{\alpha}
$$

and therefore

$$
\mathcal{D}^{\infty}\left(d \pi\left(X_{0}\right)\right)=\left\{v \in \mathcal{H}:(\forall n \in \mathbb{N}) \sum_{\alpha}\left|\alpha\left(X_{0}\right)\right|^{n}\left\|v_{\alpha}\right\|^{2}<\infty\right\},
$$


so that

$$
\mathcal{D}^{\omega}\left(d \pi\left(X_{0}\right)\right)=\left\{v \in \mathcal{H}:(\exists t>0) \sum_{\alpha} e^{t \alpha\left(X_{0}\right)}\left\|v_{\alpha}\right\|^{2}<\infty\right\}
$$

Lemma 2.1. For $X \in z(\mathfrak{f})$ the space $\mathcal{D}^{\infty}(d \pi(X))$ is K-invariant.

Proof. For $g \in G$ and $X \in g$ we have $d \pi(\operatorname{Ad}(g) . X)=\pi(g) d \pi(X) \pi(g)^{-1}$; hence

$$
\mathcal{D}^{\infty}(d \pi(\operatorname{Ad}(g) \cdot X))=\pi(g) \cdot \mathcal{D}^{\infty}(d \pi(X)) .
$$

The lemma is a direct consequence of this observation.

Lemma 2.2. Let $X_{0} \in i C_{\max }^{0}$. Then, for each $Y \in \mathrm{t}$, there exist $C, D>0$ such that for all $\alpha \in \mathcal{P}_{\lambda}$ we have $|\alpha(Y)| \leq C\left|\alpha\left(X_{0}\right)\right|+D$.

Proof. First we recall that

$$
\mathcal{P}_{\lambda} \subseteq \operatorname{conv}\left(\mathcal{W}_{\mathrm{f}} \cdot \lambda\right)-\operatorname{cone}\left(\Delta_{p}^{+}\right)
$$

(cf. [9, Proposition 2.3]). Let $C_{1}>0$ be such that $|\beta(Y)| \leq C_{1}\left|\beta\left(X_{0}\right)\right|=C_{1} \beta\left(X_{0}\right)$ holds, for all $\beta \in \Delta_{p}^{+}$, and hence for all $\beta \in \operatorname{cone}\left(\Delta_{p}^{+}\right)$. For $\alpha=\alpha_{0}-\beta$, $\alpha_{0} \in \operatorname{conv}\left(\mathcal{W}_{\mathrm{f}} . \lambda\right), \beta \in \operatorname{cone}\left(\Delta_{p}^{+}\right)$we then obtain $\beta\left(X_{0}\right)=\left(\alpha_{0}-\alpha\right)\left(X_{0}\right) \leq\left|\alpha\left(X_{0}\right)\right|+C_{2}$, where $C_{2}=\max \left|\left\langle\mathcal{W}_{f} \cdot \lambda, X_{0}\right\rangle\right|$. Now

$$
|\alpha(Y)| \leq\left|\alpha_{0}(Y)\right|+|\beta(Y)| \leq \max \left|\left\langle\mathcal{W}_{\mathfrak{f}} . \lambda, Y\right\rangle\right|+C_{1} \beta\left(X_{0}\right) \leq C_{3}+C_{1}\left(\left|\alpha\left(X_{0}\right)\right|+C_{2}\right),
$$

and this proves the lemma.

Lemma 2.3. For each $Y \in \mathrm{t}$ and $X_{0} \in i C_{\max }^{0}$ we have $\mathcal{D}^{\infty}\left(d \pi\left(X_{0}\right)\right) \subseteq \mathcal{D}^{\infty}(d \pi(Y))$.

Proof. We choose $C, D>0$ according to Lemma 2.2. For $v=\sum_{\alpha} v_{\alpha}$ $\in \mathcal{D}^{\infty}\left(d \pi\left(X_{0}\right)\right)$ we then have

$$
\begin{aligned}
\sum_{\alpha}|\alpha(Y)|^{n}\left\|v_{\alpha}\right\|^{2} & \leq \sum_{\alpha}\left(C\left|\alpha\left(X_{0}\right)\right|+D\right)^{n}\left\|v_{\alpha}\right\|^{2} \\
& =\sum_{\alpha} \sum_{k=0}^{n}\left(\begin{array}{l}
n \\
k
\end{array}\right) C^{k} D^{n-k}\left|\alpha\left(X_{0}\right)\right|^{k}\left\|v_{\alpha}\right\|^{2} \\
& =\sum_{k=0}^{n}\left(\begin{array}{l}
n \\
k
\end{array}\right) C^{k} D^{n-k} \sum_{\alpha}\left|\alpha\left(X_{0}\right)\right|^{k}\left\|v_{\alpha}\right\|^{2}<\infty .
\end{aligned}
$$

Proposition 2.4. If $X_{0}, X_{1} \in i C_{\max }^{0}$, then $\mathcal{D}^{\infty}\left(d \pi\left(X_{0}\right)\right)=\mathcal{D}^{\infty}\left(d \pi\left(X_{1}\right)\right)$.

Proof. This is an immediate consequence of Lemma 2.3.

According to Proposition 2.4, we may further assume that the element $X_{0} \in i C_{\max }^{0}$ is contained in $i_{z}(\mathfrak{f})$. In this case the following lemma provides some 
additional information. In the following we shall write $Z^{*}:=-\bar{Z}$ for elements $Z \in \mathfrak{g}_{\mathbb{C}}$, where $Z \mapsto \rightarrow \bar{Z}$ is the complex conjugation with respect to the real form $\mathrm{g}$. We extend the map $Z \rightarrow Z^{*}$ to an antilinear involutive antiautomorphism $D \rightarrow D^{*}$ of the enveloping algebra $\mathcal{U}\left(\mathfrak{g}_{\mathbb{C}}\right)$.

Lemma 2.5. If $X_{0} \in i\left(C_{\max }^{0} \cap z(\mathfrak{f})\right)$, then for each $Y \in \mathfrak{f}$ we have $\mathcal{D}^{\infty}\left(d \pi\left(X_{0}\right)\right) \subseteq \mathcal{D}^{\infty}(d \pi(Y))$.

Proof. In view of $\mathfrak{f}=\operatorname{Ad}(K)$.t, the assertion follows from Lemma 2.3 and Lemma 2.1.

Lemma 2.5 implies in particular that

$$
\mathcal{H}^{\infty}=\bigcap_{Y \in \mathfrak{g}_{\mathbb{C}}} \mathcal{D}^{\infty}(d \pi(Y))=\mathcal{D}^{\infty}\left(d \pi\left(X_{0}\right)\right) \cap \bigcap_{\alpha \in \Delta_{p}^{+}} \bigcap_{Y \in \mathrm{g}_{\mathbb{C}}^{\alpha}} \mathcal{D}^{\infty}(d \pi(Y)) .
$$

Now we deal with the different types of root spaces. Let $\alpha \in \Delta^{+}$and

$$
\mathfrak{g}_{\alpha}(Z):=\operatorname{span}_{\mathbb{R}}\left\{Z-Z^{*}, i\left(Z+Z^{*}\right)\right\}+\mathrm{t} \subseteq \mathfrak{g} .
$$

Then $W_{\max } \cap \mathfrak{g}_{\alpha}(Z)$ is an elliptic cone with interior points. Since $\pi_{\lambda}$ is a trace class representation of the maximal semigroup $S=G \exp \left(i W_{\max }^{0}\right)$ (cf. [10]), the restriction to the subgroup $G_{\alpha}(Z):=\left\langle\exp \mathfrak{g}_{\alpha}(Z)\right\rangle \subseteq G$ decomposes as a direct sum of holomorphic representations of the semigroup $S_{\alpha}(Z):=G_{\alpha}(Z) \exp \left(i\left(W_{\max }^{0} \cap \mathfrak{g}_{\alpha}(Z)\right)\right)$ with finite multiplicities (cf. [12, Theorem III.3.21]). Furthermore each highest weight $\mu$ occurring in $L(\lambda)$ is contained in $\mathcal{P}_{\lambda}$, and from (2.1) we conclude that for each $Y \in i C_{\max }$

$$
\mu(Y) \leq \sup \left\langle\mathcal{W}_{f} \cdot \lambda, Y\right\rangle
$$

For $Z \in \mathfrak{g}_{\mathbb{C}}^{\alpha}, \alpha \in \Delta_{p}^{+}$, we further have

$$
\left[Z^{*}, Z\right]=i[i \bar{Z}, Z] \in i C_{\min } \subseteq i C_{\max } .
$$

Now the condition $\lambda \in-\left(i C_{\min }\right)^{\star}$ that is necessary for unitarizability (cf. [10] or [12, Chapter IX.2]) and (2.1) together imply that

$$
\mu\left(\left[Z^{*}, Z\right]\right) \leq 0
$$

for all $\mu \in \mathcal{P}_{\lambda}$.

Lemma 2.6. For each $Z \in \mathfrak{g}_{\mathbb{C}}^{\alpha}$ there exists $Y \in$ it such that

$$
\Omega:=-Z^{*} Z-Z Z^{*}+Y\left[Z^{*}, Z\right] \in \mathcal{U}\left(g_{\mathbb{C}}\right)
$$

is central in $\mathcal{U}\left(\mathfrak{g}_{\alpha}(Z)_{\mathbb{C}}\right)$. For $\alpha \in \Delta_{p}^{+}$we can choose $Y \in i C_{\max }$ and for $\alpha \in \Delta_{r}^{+}$we can even choose $Y \in i C_{\max } \cap z(\mathfrak{f})$. Moreover we have

$$
\Omega=(1+Y)\left[Z^{*}, Z\right]-2 Z^{*} Z=(Y-\mathbf{1})\left[Z^{*}, Z\right]-2 Z Z^{*} .
$$


Proof. It is clear that $\left[\mathrm{t}_{\mathbb{C}}, \Omega\right]=\{0\}$. Furthermore we have

$$
\begin{aligned}
{[Z, \Omega] } & =-\left[Z, Z^{*}\right] Z-Z\left[Z, Z^{*}\right]+[Z, Y]\left[Z^{*}, Z\right]+Y\left[Z,\left[Z^{*}, Z\right]\right] \\
& =\left[\left[Z^{*}, Z\right], Z\right]+2 Z\left[Z^{*}, Z\right]-\alpha(Y) Z\left[Z^{*}, Z\right]-\alpha\left(\left[Z^{*}, Z\right]\right) Y Z \\
& =\alpha\left(\left[Z^{*}, Z\right]\right) Z+(2-\alpha(Y)) Z\left[Z^{*}, Z\right]-\alpha\left(\left[Z^{*}, Z\right]\right)[Y, Z]-\alpha\left(\left[Z^{*}, Z\right]\right) Z Y \\
& =\alpha\left(\left[Z^{*}, Z\right]\right) Z+(2-\alpha(Y)) Z\left[Z^{*}, Z\right]-\alpha\left(\left[Z^{*}, Z\right]\right) \alpha(Y) Z-\alpha\left(\left[Z^{*}, Z\right]\right) Z Y \\
& =\alpha\left(\left[Z^{*}, Z\right]\right)(1-\alpha(Y)) Z+Z\left((2-\alpha(Y))\left[Z^{*}, Z\right]-\alpha\left(\left[Z^{*}, Z\right]\right) Y\right) .
\end{aligned}
$$

For this expression to vanish it is necessary and sufficient that

$$
\alpha\left(\left[Z^{*}, Z\right]\right)(1-\alpha(Y))=0 \quad \text { and } \quad(2-\alpha(Y))\left[Z^{*}, Z\right]=\alpha\left(\left[Z^{*}, Z\right]\right) Y .
$$

If $\alpha\left(\left[Z^{*}, Z\right]\right) \neq 0$, then we put $Y=\frac{1}{2} \check{\alpha} \in \mathbb{R}\left[Z^{*}, Z\right]$. Note that $\alpha(Y)=1$, which means that $\left[Z^{*}, Z\right]=\alpha\left(\left[Z^{*}, Z\right]\right) Y$ and hence that (2.5) is satisfied. For $\alpha \in \Delta_{p}^{+}$we further see that $\check{\alpha} \in i C_{\max }$ follows from (2.3).

If $\alpha\left(\left[Z, Z^{*}\right]\right)=0$, then we choose $Y \in i C_{\max }$ with $\alpha(Y)=2$, which is possible for $\alpha \in \Delta_{r}^{+}$because $i C_{\max }$ is the dual cone $\Delta_{p}^{\star}$ of $\Delta_{p}^{+}$. Since $z(\mathfrak{f})$ intersects the interior of the cone $C_{\max }$, we even see that $Y$ can be chosen in $i z(\mathfrak{f})$. It is clear that $(2.5)$ is satisfied in this case. This shows that in any case a suitable choice for $Y$ yields $[\Omega, Z]=0$. Hence $\left[\Omega, Z^{*}\right]=\left[Z, \Omega^{*}\right]^{*}=[Z, \Omega]^{*}=0$ follows from

$$
\Omega^{*}=-Z^{*} Z-Z Z^{*}+\left[Z^{*}, Z\right]^{*} Y^{*}=-Z^{*} Z-Z Z^{*}+Y\left[Z^{*}, Z\right]=\Omega .
$$

Thus $\left[\Omega, t_{\mathbb{C}}+\mathbb{C} Z+\mathbb{C} Z^{*}\right]=\{0\}$ and therefore $\Omega$ is central in $\mathcal{U}\left(\mathbb{C} Z+\mathbb{C} Z^{*}+\mathfrak{t}_{\mathbb{C}}\right)$.

For the last statement we note that

$$
\Omega=-Z^{*} Z-Z Z^{*}+Y\left[Z^{*}, Z\right]=\left[Z^{*}, Z\right]-2 Z^{*} Z+Y\left[Z^{*}, Z\right]=(1+Y)\left[Z^{*}, Z\right]-2 Z^{*} Z
$$

and

$$
\Omega=-Z^{*} Z-Z Z^{*}+Y\left[Z^{*}, Z\right]=\left[Z, Z^{*}\right]-2 Z Z^{*}+Y\left[Z^{*}, Z\right]=(Y-\mathbf{1})\left[Z^{*}, Z\right]-2 Z Z^{*} .
$$

REMARK 2.7. We note that for non-compact simple roots we find a choice of $Z$ with $\left[Z^{*}, Z\right]=\check{\alpha}$, where $\alpha(\check{\alpha})=2$. Then $Y\left[Z^{*}, Z\right]=\frac{1}{2} \check{\alpha}^{2}$. If $\alpha$ is compact simple, then a suitable choice of $Z$ leads to $\left[Z^{*}, Z\right]=-\check{\alpha}$ and we get $Y\left[Z^{*}, Z\right]=-\frac{1}{2} \check{\alpha}^{2}$.

The strategy to obtain estimates for the elements in the root spaces will be to analyze the irreducible subrepresentations for $G_{\alpha}(Z)$, respectively $S_{\alpha}(Z)$, quite explicitly, and then show that the result we are heading for holds for this reduced case.

If $v=\sum_{\beta} v_{\beta} \in \mathcal{H}_{\lambda}$ is a vector decomposed according to the weight decomposition, then $Z . v=\sum_{\beta} Z . v_{\beta}$ is the weight decomposition of $Z . v$ and similarly for $Z^{*} . v$. Hence

$$
\|Z . v\|^{2}=\sum_{\beta}\left\|Z . v_{\beta}\right\|^{2}
$$

so that we shall need estimates of the type 


$$
\left\|Z . v_{\beta}\right\|^{2} \leq C(Z, \beta)\left\|v_{\beta}\right\|^{2} \quad \text { and } \quad\left\|Z^{*} \cdot v_{\beta}\right\|^{2} \leq C^{*}(Z, \beta)\left\|v_{\beta}\right\|^{2} .
$$

The following lemma shows that, for $\alpha \in \Delta_{p}^{+}$, it suffices to obtain estimates for the action of $Z^{*}$.

LeMma 2.8. For a weight vector $v_{\beta} \in L(\lambda)^{\beta}$ and $Z \in \mathfrak{g}_{\mathbb{C}}^{\alpha}, \alpha \in \Delta_{p}^{+}$, we have $\left\|Z . v_{\beta}\right\|^{2} \leq\left\|Z^{*} . v_{\beta}\right\|^{2}$.

Proof. In view of (2.4), we have

$$
\left\|Z \cdot v_{\beta}\right\|^{2}-\left\|Z^{*} \cdot v_{\beta}\right\|^{2}=\left\langle\left(Z^{*} Z-Z Z^{*}\right) \cdot v_{\beta}, v_{\beta}\right\rangle=\left\langle\left[Z^{*}, Z\right] \cdot v_{\beta}, v_{\beta}\right\rangle=\beta\left(\left[Z^{*}, Z\right]\right)\left\|v_{\beta}\right\|^{2} \leq 0 .
$$

The following exact formula will be the key to the specific estimates.

Lemma 2.9. For any weight vector $v_{\mu} \in \operatorname{ker} d \pi(Z)$ and $v_{\beta}=\left(Z^{*}\right)^{n} \cdot v_{\mu}$ we have

$$
\left\|Z \cdot v_{\beta}\right\|^{2}=\frac{1}{2}\left((1+\beta(Y)) \beta\left(\left[Z^{*}, Z\right]\right)-(1+\mu(Y)) \mu\left(\left[Z^{*}, Z\right]\right)\right)\left\|v_{\beta}\right\|^{2}
$$

and

$$
\left\|Z^{*} \cdot v_{\beta}\right\|^{2}=\frac{1}{2}\left((\beta(Y)-1) \beta\left(\left[Z^{*}, Z\right]\right)-(1+\mu(Y)) \mu\left(\left[Z^{*}, Z\right]\right)\right)\left\|v_{\beta}\right\|^{2}
$$

Proof. According to Lemma 2.6 and $Z . v_{\mu}=0$, we have

$$
\Omega \cdot v_{\mu}=(\mathbf{1}+Y)\left[Z^{*}, Z\right] \cdot v_{\mu}=(1+\mu(Y)) \mu\left(\left[Z^{*}, Z\right]\right) v_{\mu} .
$$

For $v_{\beta}=\left(Z^{*}\right)^{n} \cdot v_{\mu}$ we now obtain from the fact that $\Omega$ commutes with $Z$

$$
\begin{aligned}
2\left\|Z \cdot v_{\beta}\right\|^{2} & =2\left\langle Z^{*} Z \cdot v_{\beta}, v_{\beta}\right\rangle=\left\langle\left((1+Y)\left[Z^{*}, Z\right]-\Omega\right) \cdot v_{\beta}, v_{\beta}\right\rangle \\
& =\left((1+\beta(Y)) \beta\left(\left[Z^{*}, Z\right]\right)-(1+\mu(Y)) \mu\left(\left[Z^{*}, Z\right]\right)\right)\left\|v_{\beta}\right\|^{2}
\end{aligned}
$$

and

$$
\begin{aligned}
2\left\|Z^{*} \cdot v_{\beta}\right\|^{2} & =2\left\langle Z Z^{*} \cdot v_{\beta}, v_{\beta}\right\rangle=\left\langle\left((Y-\mathbf{1})\left[Z^{*}, Z\right]-\Omega\right) \cdot v_{\beta}, v_{\beta}\right\rangle \\
& =\left((\beta(Y)-1) \beta\left(\left[Z^{*}, Z\right]\right)-(1+\mu(Y)) \mu\left(\left[Z^{*}, Z\right]\right)\right)\left\|v_{\beta}\right\|^{2} .
\end{aligned}
$$

Lemma 2.10. For each $n \in \mathbb{N}, Z \in \mathfrak{g}_{\mathbb{C}}^{\alpha}, \alpha \in \Delta_{p}$, and $Y$ as in Lemma 2.6, there exists a polynomial $P$ with non-negative coefficients such that for any $\mathfrak{t}_{\mathbb{C}}$-weight vector $v_{\beta} \in L(\lambda)^{\beta}$ we have

$$
\left\|Z^{n} \cdot v_{\beta}\right\|^{2} \leq P(|\beta(Y)|)\left\|v_{\beta}\right\|^{2}
$$


Proof. Let $Z \in \mathfrak{g}_{\mathbb{C}}^{\alpha}$ and $\alpha \in \Delta_{p}^{+}$. To prove the lemma we have to estimate the expressions $\left\|Z^{n} \cdot v_{\beta}\right\|^{2}$ and $\left\|\left(Z^{*}\right)^{n} \cdot v_{\beta}\right\|^{2}$ because, in the statement of the lemma, it is not assumed that $\alpha$ is a positive root.

Since $L(\lambda)$ decomposes as an orthogonal direct sum of highest weight modules $L(\mu)_{Z, \alpha}$ for the Lie algebra $\mathfrak{g}_{\alpha}(Z)_{\mathbb{C}}$, we only have to prove the estimates for such modules and have to check that the polynomials obtained are independent of the highest weight $\mu$ of such a submodule. We let $v_{\mu} \in \operatorname{ker} d \pi(Z)$ and $v_{\beta}=\left(Z^{*}\right)^{m} \cdot v_{\mu}$.

We first assume that $\alpha \in \Delta_{p, s}^{+}$and recall that in this case $Y=\frac{1}{2} \check{\alpha}=\frac{1}{2}\left[Z^{*}, Z\right]$ if $Z$ is normalized such that $\alpha\left(\left[Z^{*}, Z\right]\right)=2$ (Remark 2.7). From $\beta \in \mu-\mathbb{N}_{0} \alpha$ and $\alpha(\check{\alpha})=2$ we conclude with $(2.4)$ that $\beta(\check{\alpha}) \leq \mu(\check{\alpha}) \leq 0$; hence $|\beta(\check{\alpha})| \geq|\mu(\check{\alpha})|$. Thus Lemma 2.9 gives

$$
2\left\|Z^{*} \cdot v_{\beta}\right\|^{2} \leq(2+|\beta(\check{\alpha})|)|\beta(\check{\alpha})|\left\|v_{\beta}\right\|^{2} \leq(2+|\beta(\check{\alpha})|)^{2}\left\|v_{\beta}\right\|^{2} .
$$

Inductively this leads to

$$
\left\|\left(Z^{*}\right)^{n} \cdot v_{\beta}\right\|^{2} \leq \frac{1}{2^{n}} \prod_{j=1}^{n}(2-(\beta(\check{\alpha})-2(j-1)))^{2}\left\|v_{\beta}\right\|^{2} \leq \frac{1}{2^{n}}(2 n-\beta(\check{\alpha}))^{2 n}\left\|v_{\beta}\right\|^{2},
$$

and so we have an estimate of the type

$$
\left\|\left(Z^{*}\right)^{n} \cdot v_{\beta}\right\|^{2} \leq P_{1}(|\beta(Y)|)\left\|v_{\beta}\right\|^{2}
$$

where $P_{1}$ is a polynomial of degree $2 n$ with non-negative coefficients.

Using Lemma 2.8 and (2.6), we get $2\left\|Z . v_{\beta}\right\|^{2} \leq(2+|\beta(\check{\alpha})|)^{2}\left\|v_{\beta}\right\|^{2}$ and, inductively, this also leads to an estimate of the form

$$
\left\|Z^{n} \cdot v_{\beta}\right\|^{2} \leq P_{2}(|\beta(Y)|)\left\|v_{\beta}\right\|^{2} .
$$

Now we consider the case where $\alpha\left(\left[Z^{*}, Z\right]\right)=0$; i.e., $\alpha \in \Delta_{r}^{+}$. We choose $Y \in z(f)$ (Lemma 2.6). Then $\left[Z^{*}, Z\right]$ is central in g (cf. [12, Section VII.2]), so that $\beta\left(\left[Z^{*}, Z\right]\right)=\lambda\left(\left[Z^{*}, Z\right]\right)$ holds, for all $\beta \in \mathcal{P}_{\lambda}$. Moreover $\mu \in \mathcal{P}_{\lambda}$ and (2.1) show that $\beta(Y) \leq \mu(Y) \leq \lambda(Y)$. Therefore

$$
|\beta(Y)-\mu(Y)|=\mu(Y)-\beta(Y) \leq \lambda(Y)-\beta(Y) .
$$

Hence

$$
\begin{aligned}
\left\|Z^{*} \cdot v_{\beta}\right\|^{2} & =\frac{1}{2}\left((\beta(Y)-1) \beta\left(\left[Z^{*}, Z\right]\right)-(1+\mu(Y)) \mu\left(\left[Z^{*}, Z\right]\right)\right)\left\|v_{\beta}\right\|^{2} \\
& =\frac{\lambda\left(\left[Z^{*}, Z\right]\right)}{2}(\beta(Y)-\mu(Y)-2)\left\|v_{\beta}\right\|^{2} \\
& \leq \frac{\left|\lambda\left(\left[Z^{*}, Z\right]\right)\right|}{2}(\lambda(Y)-\beta(Y)+2)\left\|v_{\beta}\right\|^{2} \\
& \leq \frac{\left|\lambda\left(\left[Z^{*}, Z\right]\right)\right|}{2}(|\lambda(Y)|+|\beta(Y)|+2)\left\|v_{\beta}\right\|^{2}
\end{aligned}
$$

Now the same arguments as for $\alpha \in \Delta_{p, s}^{+}$apply and show that the inequalities 


$$
\left\|\left(Z^{*}\right)^{n} \cdot v_{\beta}\right\|^{2} \leq P_{3}(|\beta(Y)|)\left\|v_{\beta}\right\|^{2}, \quad\left\|Z^{n} \cdot v_{\beta}\right\|^{2} \leq P_{4}(|\beta(Y)|)\left\|v_{\beta}\right\|^{2}
$$

hold for polynomials $P_{3}, P_{4}$ with non-negative coefficients.

THEOREM 2.11. For each $X_{0} \in i W_{\max }^{0}$ we have $\mathcal{H}_{\lambda}^{\infty}=\mathcal{D}^{\infty}\left(d \pi\left(X_{0}\right)\right)$.

Proof. Since $W_{\max }^{0}=\operatorname{Ad}(G) \cdot C_{\max }^{0}$ ([11, Lemma II.10]), we may without loss of generality assume that $X_{0} \in i C_{\max }^{0}$. Further Proposition 2.4 shows that we may even assume that $X_{0} \in i C_{\max }^{0} \cap i_{\zeta}(\mathfrak{f})$. Then Lemma 2.5 shows that for each $Y \in \mathfrak{f}$ we have $\mathcal{D}^{\infty}\left(d \pi\left(X_{0}\right)\right) \subseteq \mathcal{D}^{\infty}(d \pi(Y))$. It remains to show that, for each $\alpha \in \Delta_{p}$ and $Z \in \mathfrak{g}_{\mathbb{C}}^{\alpha}$, we have $\mathcal{D}^{\infty}\left(d \pi\left(X_{0}\right)\right) \subseteq \mathcal{D}^{\infty}(d \pi(Z))$.

Using Lemma 2.2, we find $C, D>0$ with $|\beta(Y)| \leq C\left|\beta\left(X_{0}\right)\right|+D$, for all $\beta \in \mathcal{P}_{\lambda}$. Thus we see from Lemma 2.10 that for $v=\sum_{\alpha} v_{\beta} \in \mathcal{D}^{\infty}\left(d \pi\left(X_{0}\right)\right)$ we get

$$
\begin{aligned}
\left\|Z^{n} \cdot v\right\|^{2} & =\sum_{\beta}\left\|Z^{n} \cdot v_{\beta}\right\|^{2} \leq \sum_{\beta} P(|\beta(Y)|)\left\|v_{\beta}\right\|^{2} \\
& \leq \sum_{\beta} P\left(C\left|\beta\left(X_{0}\right)\right|+D\right)\left\|v_{\beta}\right\|^{2}<\infty .
\end{aligned}
$$

We conclude that $\mathcal{D}\left(d \pi\left(X_{0}\right)\right) \subseteq \mathcal{D}(d \pi(Z))$. This completes the proof.

\section{REFERENCES}

1. J. Faraut and A. Koranyi, Function spaces and reproducing kernels on bounded symmetric domains, J. Funct. Anal. 88 (1990), 64-89.

2. J. Faraut and A. Koranyi, Analysis on symmetric cones (Oxford University Press, 1994).

3. Harish-Chandra, Representations of semi-simple Lie groups, IV, Amer. J. Math. 77 (1955), 743-777.

4. Harish-Chandra, Representations of semi-simple Lie groups, V, VI, Amer. J. Math. 78 (1956), 1-41, 564-628.

5. J. Hilgert, K. H. Hofmann and J. D. Lawson, Lie groups, convex cones and semigroups (Oxford University Press, 1989).

6. B. Krötz, Norm estimates for unitarizable highest weight representations, Ann. Inst. Fourier (Grenoble) 49 (1999), No. 4, 1242-1264.

7. B. Krötz, K.-H. Neeb and G. Ólafsson, Spherical representations and mixed symmetric spaces, J. Representation Theory 1 (1997), 424-461.

8. K.-H. Neeb, Holomorphic representation theory II, Acta Math. 173 (1994), No. 1, $103-133$.

9. K.-H. Neeb, On the convexity of the moment mapping for a unitary highest weight representation, J. Funct. Anal. 127 (1995), No. 2, 301-325.

10. K.-H. Neeb, Coherent states, holomorphic extensions, and highest weight representations, Pacific J. Math. 174 (1996), No. 2, 497-542.

11. K.-H. Neeb, Invariant convex sets and functions in Lie algebras, Semigroup Forum 53 (1996), 230-261.

12. K.-H. Neeb, Holomorphy and convexity in Lie theory, Expositions in Mathematics No. 28 (de Gruyter, 1999). 